\title{
Monte Carlo study of kink effect in short-channel InAIAs/lnGaAs high electron mobility transistors
}

\author{
B. G. Vasallo, a) J. Mateos, D. Pardo, and T. González \\ Departamento de Física Aplicada, Universidad de Salamanca, Plaza de la Merced s/n, \\ 37008 Salamanca, Spain
}

(Received 12 February 2003; accepted 25 June 2003)

\begin{abstract}
A semiclassical two-dimensional ensemble Monte Carlo simulator is used to perform a physical microscopic analysis of the kink effect in short-channel InAlAs/InGaAs lattice-matched high electron mobility transistors (HEMTs). Due to the small band gap of InGaAs, these devices are very susceptible to suffer impact ionization processes, with the subsequent hole transport in the channel, both supposedly implicated in the kink effect and easy to be implemented in a Monte Carlo simulation. The results indicate that for high enough $V_{\mathrm{DS}}$, holes, generated by impact ionization, tend to pile up in the channel under the source side of the gate due to the attracting potential caused by the surface charge at the recess and, mostly, by the gate potential. Due to this pile up of positive charge, the potential barrier controlling the current through the channel is lowered, so that the channel is further opened and $I_{D}$ increases, leading to the well known kink effect in the currentvoltage characteristics. The microscopic understanding of this phenomenon provides valuable information to conceive the optimum fabrication process for kink-effect-free HEMTs. (C) 2003 American Institute of Physics. [DOI: 10.1063/1.1603955]
\end{abstract}

\section{INTRODUCTION}

InAlAs/InGaAs high electron mobility transistors (HEMTs) have proven a record of excellent performance for low-noise high-frequency applications (in microwave and millimeter-wave frequency ranges), and are used as active devices in high-speed integrated circuits. ${ }^{1-3}$ However, they have still some drawbacks to be eliminated, like the kink effect, i.e., an anomalous increase in the drain current $I_{D}$ at sufficiently high drain-to-source voltages $V_{\mathrm{DS}}$, which leads to a reduction in the gain and a rise in the level of noise, thus limiting the utility of these devices for microwave power applications. $^{4}$

When reducing the device dimensions to improve the frequency range of operation of HEMTs, very high electric fields appear in the gate-drain region of the device, which, jointly with the narrow band gap of InGaAs, makes this device very susceptible to impact ionization mechanisms. ${ }^{1}$ Some works suggest that impact ionization and the subsequent hole dynamics (jointly with trapping processes) can be responsible for the kink effect. ${ }^{4-10}$ However, kink phenomena are not still completely understood, specially in shortchannel HEMTs. ${ }^{4}$

Thus, the use of a microscopic approach beyond the standard drift-diffusion models typically employed to analyze this effect is highly desirable. The Monte Carlo (MC) method has been proven to be a very useful tool when dealing with problems where the understanding of the microscopic behavior of carriers is essential. ${ }^{11-14}$ The aim of this work is the development of a physical model for the kink effect in short-channel recessed $\operatorname{In}_{0.52} \mathrm{Al}_{0.48} \mathrm{As} / \mathrm{In}_{0.53} \mathrm{Ga}_{0.47} \mathrm{As}$ HEMTs. Toward this end, the microscopic transport of carri-

a)Electronic mail: a50343@usal.es ers in these devices is monitored by means of a semiclassical two-dimensional (2D) ensemble MC simulator in which both impact ionization and hole recombination are included. This approach allows one to determine the origin and magnitude of the kink effect in terms of internal quantities (like electron and hole concentrations and potential profiles), so that a complete physical understanding of the kink effect is achieved, thus providing some guidelines to be followed in the fabrication process of HEMTs in order to improve their immunity to kink effect.

The article is organized as follows. In Sec. II, the physical model is detailed. The main results of our simulations and their discussion are provided in Sec. III. Finally, in Sec. IV, we draw the most important conclusions of this work.

\section{PHYSICAL MODEL}

As mentioned before, for the calculations, we make use of an ensemble MC simulator self-consistently coupled with a 2D Poisson solver which incorporates all the processes at the origin of the kink effect. The structure under analysis is a $100 \mathrm{~nm}$ T-gate recessed HEMT (Fig. 1), and consists of a InP substrate (not simulated), a $200 \mathrm{~nm} \operatorname{In}_{0.52} \mathrm{Al}_{0.48}$ As buffer followed by a $25 \mathrm{~nm}$ thick $\mathrm{In}_{0.53} \mathrm{Ga}_{0.47}$ As channel, three layers of $\mathrm{In}_{0.52} \mathrm{Al}_{0.48} \mathrm{As}$ (a $5 \mathrm{~nm}$ spacer, a $5 \times 10^{12} \mathrm{~cm}^{-2}, \delta$-doped layer modeled as a $5 \mathrm{~nm}$ layer doped at $N_{D}=10^{19} \mathrm{~cm}^{-3}$ and a $10 \mathrm{~nm}$ Schottky layer), and, finally, a $10 \mathrm{~nm}$ thick $\mathrm{In}_{0.53} \mathrm{Ga}_{0.47}$ As cap layer $\left(N_{D}=5 \times 10^{18} \mathrm{~cm}^{-3}\right)$. The parameters for electrons in the involved materials can be found in Ref. 11, while details of the static characteristics and noise behavior of this device for low applied voltages (in absence of impact ionization) can be found in Refs. 12 and 13, in which the agreement between the results of the simulations and the experimental measurements confirm the validity of 


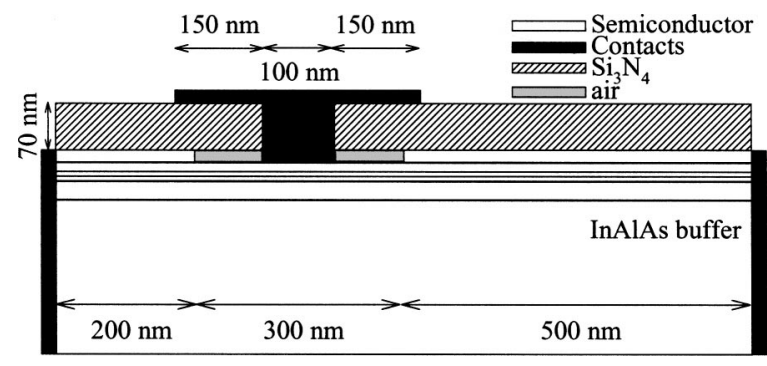

FIG. 1. Schematic drawing of the HEMT topology used in the simulations.

the model. In particular, the value adopted for the surface charge at the bottom of the recess, which, as explained in the following, may have a significant importance in the kink effect, is $\sigma / q=4.3 \times 10^{12} \mathrm{~cm}^{-2}$, a value that leads to a good agreement between simulated and experimental measurements. ${ }^{11}$

The impact ionization of electrons, which occurs in the $\Gamma$ valley and leads to the appearance of holes, is included in the MC simulations by using the Keldysh approach, ${ }^{15}$ where the probability per unit time of having an impact ionization event is given by $P(E)=S\left(E-E_{\mathrm{th}} / E_{\mathrm{th}}\right)^{2}$ if $E>E_{\mathrm{th}}$, and $P(E)=0$ if $E<E_{\mathrm{th}}, E$ being the electron kinetic energy in the $\Gamma$ valley, $E_{\mathrm{th}}$ is the ionization threshold energy, and $S$ is a measure of the softness or hardness of the threshold. $E_{\mathrm{th}}$ and $S$ are considered as adjustable parameters to reproduce the ionization coefficients (number of impact ionization events that occur per unit length) measured in bulk materials. ${ }^{16-19}$ Figure 2 shows the experimental and simulated values of the impact ionization coefficient for $\mathrm{In}_{0.53} \mathrm{Ga}_{0.47} \mathrm{As}$ (channel material) as a function of the inverse of the electric field. There is a large dispersion in the experimental measurements and, moreover, for moderate electric fields $(80-150 \mathrm{kV} / \mathrm{cm})$, at which the device usually works, no measurements are available. In our simulations, we will consider the two sets of parameters reported in Fig. 2: $E_{\mathrm{th}}=0.86 \mathrm{eV}, S=10^{12} \mathrm{~s}^{-1}$, and $E_{\mathrm{th}}=0.8 \mathrm{eV}, S=2 \times 10^{12} \mathrm{~s}^{-1}$. The agreement of the simulated ionization coefficients with the experimental data is reasonable in both cases. Even if the first set of parameters is more realistic and fits better the experimental values, we will mainly use the second case, since the central processing unit (CPU) time required to reduce the uncertainty of the results is more affordable because of the larger number of ionization events taking place in the devices. From each impact ionization occurrence, an electron in the $\Gamma$ valley and a hole in the heavy-hole $(\mathrm{HH})$ band emerge, while the electron

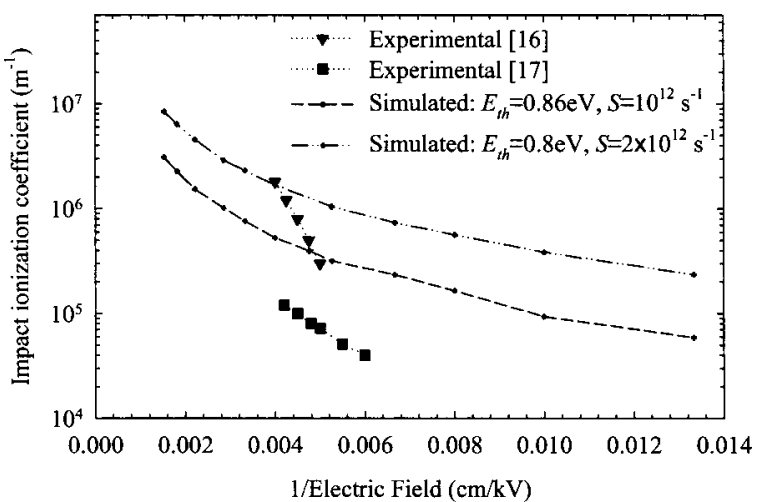

FIG. 2. Experimental (see Refs. 16 and 17) and simulated impact ionization coefficient vs inverse of electric field in $\mathrm{In}_{0.53} \mathrm{Ga}_{0.47} \mathrm{As}$.

originating the ionization process remains in the $\Gamma$ valley. We have verified that the hole impact ionization is negligible for the considered applied voltages.

With respect to the model used for hole dynamics (the main aspect of our HEMT simulator in order to include impact ionization processes), a typical spherical and nonparabolic valence-band structure is considered, including three sub-bands: $\mathrm{HH}$ and light-hole (LH) bands, degenerated at $\mathbf{k}=0$ and characterized by a different curvature in $\mathbf{k}$ space, and a third split-off hole ( $\mathrm{SOH})$ band, in which the band warping is accounted for by the use of approximated overlap functions. ${ }^{20}$ Ionized impurity, acoustic, polar, and nonpolar optical phonon scattering mechanisms are considered for holes. ${ }^{20,21}$ The hole physical parameters used in the simulations are reported in Table I. They have been obtained by interpolating between the values of the corresponding binary materials. ${ }^{22-24}$ Another important process that is necessary to take into account for a proper analysis of the kink effect is hole recombination. ${ }^{4,8}$ Toward this end, we use a simple model in which hole recombination is considered to take place with a characteristic time $\tau_{\text {rec }}$ (i.e., with a probability $\left.1 / \tau_{\text {rec }}\right)$. We will perform simulations with $\tau_{\text {rec }}$ ranging between 0.01 and $1.0 \mathrm{~ns}$, in order to study its influence on the kink properties. The value of $\tau_{\text {rec }}$ constitutes a severe limitation for the simulation time, since hole recombination is the process with the longest characteristic time among those involved in the system under analysis. Thus, simulation times of at least several times $\tau_{\text {rec }}$ are required to achieve correct stationary results.

TABLE I. Physical parameters of holes in $\operatorname{In}_{0.53} \mathrm{Ga}_{0.47} \mathrm{As}$ and $\operatorname{In}_{0.52} \mathrm{Al}_{0.48} \mathrm{As}$.

\begin{tabular}{|c|c|c|c|c|c|c|}
\hline Parameter & \multicolumn{3}{|c|}{$\mathrm{In}_{0.53} \mathrm{Ga}_{0.47} \mathrm{As}$} & \multicolumn{3}{|c|}{$\mathrm{In}_{0.52} \mathrm{Al}_{0.48} \mathrm{As}$} \\
\hline GAP $(e V)$ & & 0.75 & & & 1.45 & \\
\hline Optical deformation potential $\left(10^{22} \mathrm{eV}^{2} / \mathrm{m}^{2}\right)$ & & 5.75 & & & 2.6 & \\
\hline Acoustic deformation potential $(\mathrm{eV})$ & & 4.95 & & & 4.95 & \\
\hline & $\mathrm{HH}$ & $\mathrm{LH}$ & $\mathrm{SOH}$ & $\mathrm{HH}$ & LH & $\mathrm{SOH}$ \\
\hline Effective mass $\left(\mathrm{m}^{*} / \mathrm{m}_{0}\right)$ & 0.4828 & 0.0479 & 0.1358 & 0.7012 & 0.2002 & 0.2072 \\
\hline Nonparabolicity $(1 / \mathrm{eV})$ & 1.2 & 0.9 & 0.8 & 1.05 & 1.0 & 0.68 \\
\hline Energy level from $\mathrm{HH}(\mathrm{eV})$ & 0.0 & 0.0 & 0.15 & 0.0 & 0.0 & 0.15 \\
\hline
\end{tabular}



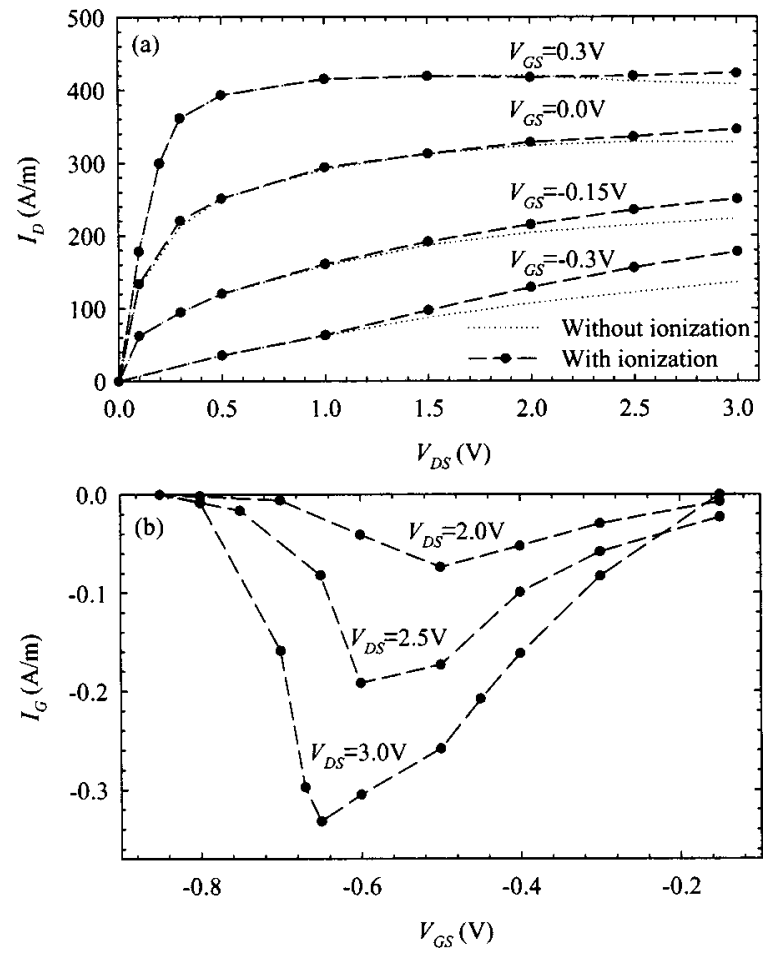

FIG. 3. Output characteristics and $I_{G}$ versus $V_{\mathrm{GS}}$ curves of the HEMT for $E_{\text {th }}=0.86 \mathrm{eV}, S=10^{12} \mathrm{~s}^{-1}$, and $\tau_{\text {rec }}=1.0 \mathrm{~ns}$.

\section{RESULTS}

Figure 3 shows the HEMT output characteristics in presence and absence of impact ionization for $E_{\mathrm{th}}=0.86 \mathrm{eV}$ and $S=10^{12} \mathrm{~s}^{-1}$, and considering $\tau_{\mathrm{rec}}=1.0 \mathrm{~ns}$ as in Refs. 4 and 8 (quite realistic parameters). A notable increase of $I_{D}$ takes place starting from a value of $V_{D S}$ high enough for the onset of impact ionization. This value of $V_{D S}$ is larger the higher $V_{G S}$ is, while the increase of $I_{D}$ is lower. This behavior is in agreement with the experimental measurements of kink effect, ${ }^{4}$ and it is consistent with the lower gate-drain field that appears in the device for fixed $V_{\mathrm{DS}}$ when $V_{\mathrm{GS}}$ increases, which leads to lower electron energy and, thus, lower impact ionization rate. In Fig. 3(b), the characteristic "bell" shape in the $I_{G}$ versus $V_{\mathrm{GS}}$ curves, signature of impact ionization in field effect transistors, can be observed. ${ }^{4,6,7}$ The gate current, being due to holes generated by impact ionization in the channel able to reach this terminal, shows this behavior as a result of the interplay between the higher concentration and the lower energy of electrons in the channel appearing when $V_{\mathrm{GS}}$ increases. At low $V_{\mathrm{GS}}$, the number of electrons crossing the channel is low due to the action of the gate (the channel is pinched off), while for high $V_{\mathrm{GS}}$, the gate-to-drain potential is lower (and thus also the kinetic energy of electrons) than for intermediate $V_{\mathrm{GS}}$. In both situations, the impact ionization events and, consequently, the number of holes that reach the gate, decrease with respect to the range of intermediate $V_{\mathrm{GS}}$, for which $I_{G}$ takes the highest values. We must point out that gate current due to electron tunneling is not considered in our model. Thus, if a comparison of $I_{G}$ with experimental measurements is to be made, the gate electron tunnel current should be added to the values plotted in Figs. 3(b) and 4(b) corresponding just to the hole contribution.
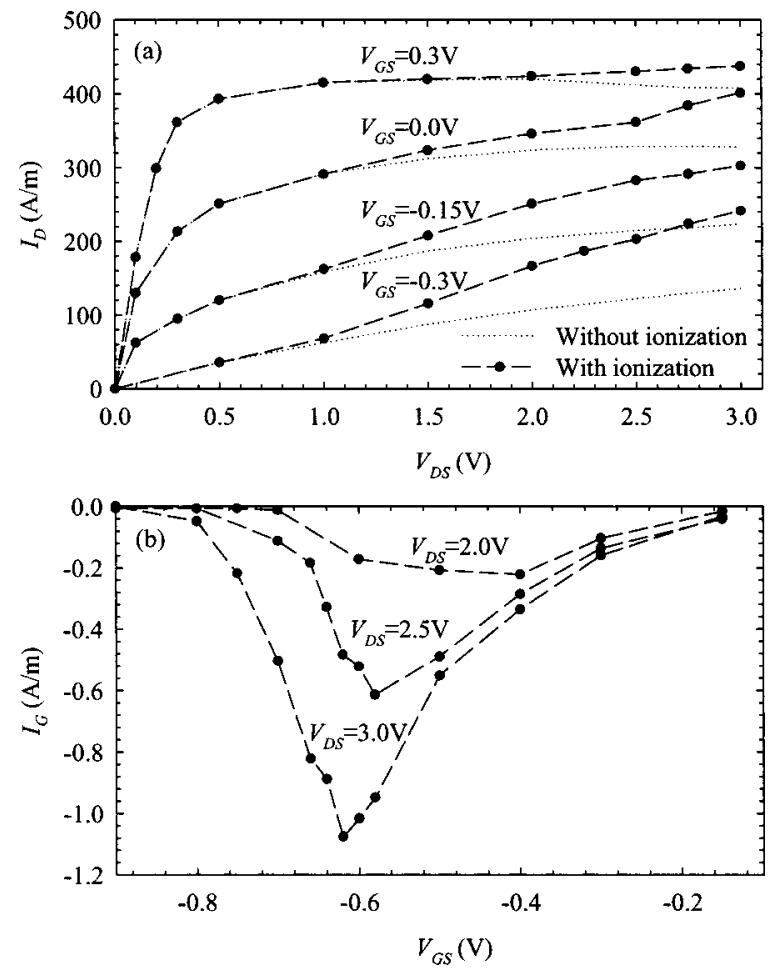

FIG. 4. Output characteristics and $I_{G}$ versus $V_{\mathrm{GS}}$ curves of the HEMT for $E_{\mathrm{th}}=0.8 \mathrm{eV}, S=2 \times 10^{12} \mathrm{~s}^{-1}$, and $\tau_{\text {rec }}=0.1 \mathrm{~ns}$.

For the realistic values of $E_{\mathrm{th}}, S$, and $\tau_{\text {rec }}$, considered in Fig. 3, the simulated time for every point of the $I_{D}-V_{\mathrm{DS}}$ and $I_{G}-V_{\mathrm{GS}}$ curves is $5.0 \mathrm{~ns}$, which requires a very long $\mathrm{CPU}$ time. Thus, $E_{\mathrm{th}}=0.8 \mathrm{eV}$ and $S=2 \times 10^{12} \mathrm{~s}^{-1}$, providing higher (but still reasonable) values for the impact ionization coefficient [Fig. 2], jointly with a shorter recombination time, will be used in the following to achieve affordable computation times. Figure 4 shows the output characteristics and the $I_{G}$ versus $V_{\mathrm{GS}}$ curves when this impact ionization coefficient and a recombination time of 0.1 ns (to counterbalance the effect of the higher ionization coefficient) are considered. The qualitative behavior of the kink is similar to that shown in Fig. 3, but the effect is stronger, as evidenced by the more pronounced increase of $I_{D}$. Under these conditions, when the kink is more explicit, it is easier to detect the origin of this effect and analyze it in detail, as it is our aim. It is important to remark that Gunn oscillations (with frequencies above $100 \mathrm{GHz}$ ) are found for the highest values of $V_{G S}$ and $V_{D S}$ reported in Figs. 3 and 4, where the values shown for $I_{D}$ are the result of a long enough time average.

MC simulations provide an insight into the microscopic processes taking place inside the devices, in terms of which the kink effect can be explained. Initially, the influence of $\tau_{\text {rec }}$ will be analyzed. For this sake, we will focus our attention on the simulations performed with the impact ionization parameters $E_{\mathrm{th}}=0.8 \mathrm{eV}$ and $S=2 \times 10^{12} \mathrm{~s}^{-1}$, for which an appreciable kink effect is observed even for low values of $\tau_{\text {rec }}$ (in the more realistic case of Fig. 3, the kink is significant only for $\tau_{\text {rec }}=1.0 \mathrm{~ns}$ ). Figure 5 shows the profiles along the channel of several internal quantities of interest for the bias point $V_{\mathrm{DS}}=3.0 \mathrm{~V}, V_{\mathrm{GS}}=-0.3 \mathrm{~V}$, and three values of $\tau_{\text {rec }}(1.0 \mathrm{~ns}, 0.1 \mathrm{~ns}$, and $0.01 \mathrm{~ns})$. The results in the absence of 


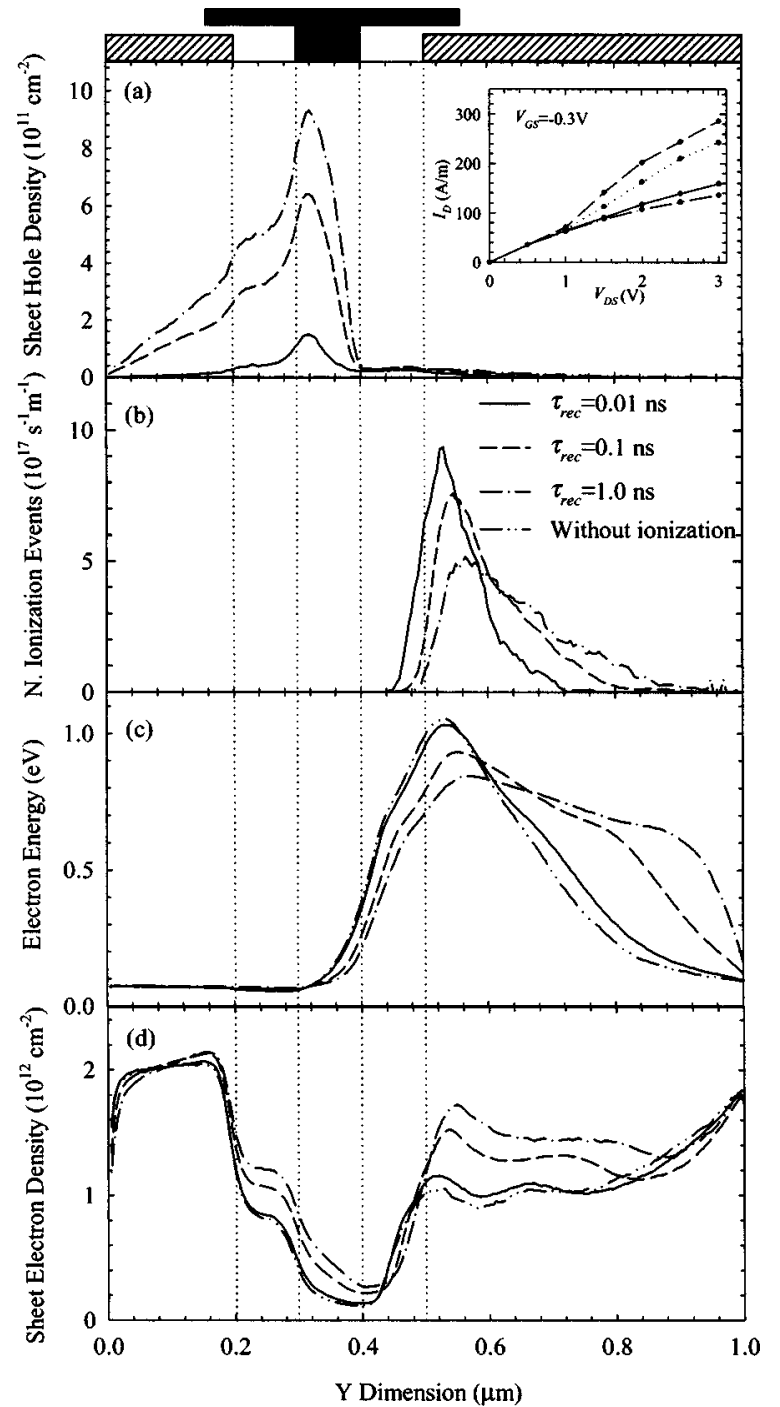

FIG. 5. Profiles along the channel of: (a) Hole sheet density (inset, output characteristics for $V_{G S}=-0.3$ ), (b) impact ionization events per unit time and depth, (c) electron energy, (d) electron sheet density for $V_{\mathrm{DS}}=3.0 \mathrm{~V}$, $V_{\mathrm{GS}}=-0.3 \mathrm{~V}, E_{\mathrm{th}}=0.8 \mathrm{eV}, S=2 \times 10^{12} \mathrm{~s}^{-1}$, and different values of $\tau_{\mathrm{rec}}$. The position of the gate and the recess is also indicated.

impact ionization are also shown for comparison. As observed in Fig. 5(b), the impact ionization events occur in the drain side of the channel, since the electric field in this region and, consequently, the electron energy [Fig. 5(c)], are higher. Figure 5(a) shows that, apart from a few holes that reach the gate and source electrodes, most of the holes generated by impact ionization cross the channel and pile up under the source side of the gate (without reaching the Schottky layer because of the energy barrier present in the valence band at the heterojunction). This accumulation of holes appears due to the attracting force of the gate potential (principally) and of the negative surface charge at the recess. The increase of $I_{D}$ can be explained as a consequence of this pile up of positive charge, which lowers the potential barrier that controls the current through the channel, so that the channel is further opened, with the consequent increase in the electron density [Fig. 5(d)] and drain current. The rise of $I_{D}$ is essentially due to this enhancement in the electron flow through the channel, since the number of electrons/holes

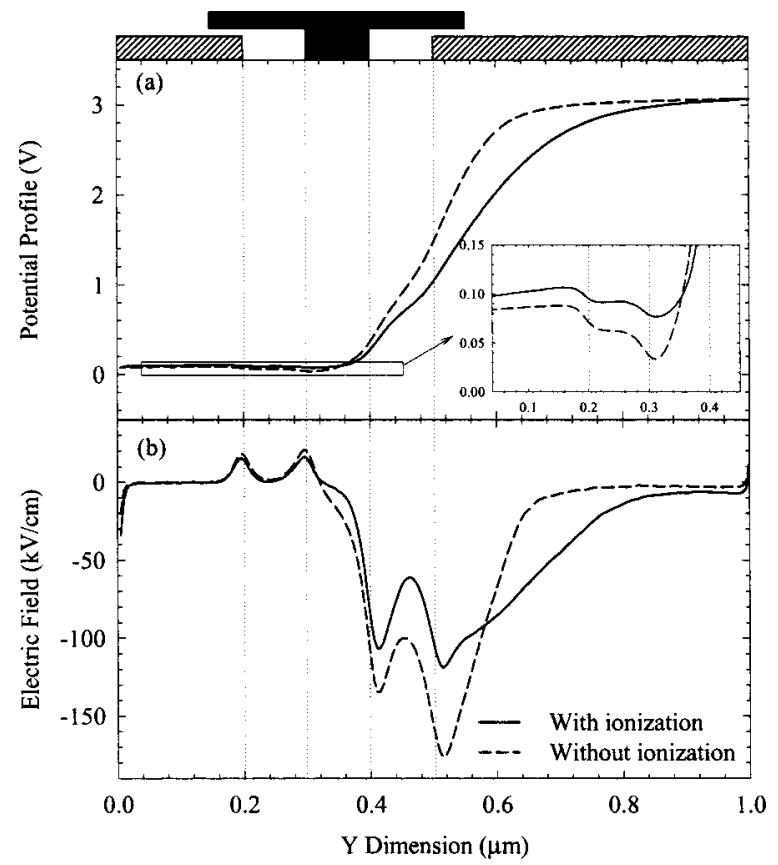

FIG. 6. (a) Potential and (b) electric-field profiles along the channel at $5 \mathrm{~nm}$ from the spacer in presence and absence of impact ionization for $V_{\mathrm{DS}}$ $=3.0 \mathrm{~V}, V_{\mathrm{GS}}=-0.3 \mathrm{~V}, E_{\mathrm{th}}=0.8 \mathrm{eV}, S=2 \times 10^{12} \mathrm{~s}^{-1}$, and $\tau_{\text {rec }}=0.1 \mathrm{~ns}$. The position of the gate and the recess is also indicated. The inset in (a) details the lowest values of the potential.

generated by impact ionization is very low so as to provide a significant contribution to $I_{D}$. Moreover, few holes reach the source contact because, as explained before, they tend to pile up under the source side of the gate, and recombine before reaching the source. The effect previously explained is more pronounced the longer $\tau_{\text {rec }}$ is, since, even if the number of impact ionization events is practically the same, the hole pile up is larger due to the longer time it takes holes to recombine [Fig. 5(a)], which leads to a stronger kink-related increase of $I_{D}$ [inset Fig. 5(a)]. In view of this result, one way of enhancing the immunity of HEMTs to the kink effect could be the use of some technological process in order to intentionally decrease $\tau_{\text {rec }}$ (for example, by locally introducing defects under the gate). However, this process has to be carefully controlled to avoid a reduction of $\tau_{\text {rec }}$ below the transit time of the electrons under the gate (of the order of $1.0 \mathrm{ps}$ ), since this would lead to a deterioration of electron mobility and population in the channel, thus degrading the performance of the HEMTs.

To illustrate in more detail the role played by the hole pile up at the gate-to-source side of the channel, Fig. 6 presents the potential profile [Fig. 6(a)] and the electric field along the channel at $5 \mathrm{~nm}$ from the spacer [Fig. 6(b)] for a bias of $V_{\mathrm{DS}}=3.0 \mathrm{~V}, V_{\mathrm{GS}}=-0.3 \mathrm{~V}$, with $\tau_{\text {rec }}=0.1 \mathrm{~ns}$, in the cases of simulations with and without impact ionization. Figure 6(b) shows that impact ionization events take place just where the maximum electric field appears, at the drain side of the recess. For this reason, the kink effect is commonly avoided (in fact, shifted to higher biasings) by enlarging the drain recess length, which leads to a lower electric field in that region. 
In the presence of impact ionization, the energy barrier controlling the passage of electrons through the channel decreases in the position of the hole pile up [Fig. 5(a)] due to the presence of the positive charge accumulation. As a consequence, more electrons coming from the source have enough energy to surpass the barrier and cross the channel, thus contributing to the enhancement of the drain current. Note that even if the difference in the potential barrier is very small, just of the order of $k_{B} T / q$ [inset Fig. 6(a)], it is enough to produce a significant variation in the number of thermal carriers coming from the source able to pass over the barrier. Additionally, due to the strong increase of the electron density in the drain side of the channel [Fig. 5(d)], the potential profile becomes smoother in this portion of the channel, so that the electric field is distributed more uniformly along the gate-drain region with respect to the case of absence of impact ionization. This effect is more pronounced the higher $\tau_{\text {rec }}$ is. Indeed, with the increase of $\tau_{\text {rec }}$, both the electron energy and the number of impact ionization mechanisms are lower under the drain side of the recess but higher in the region near the drain contact [Figs. 5(b) and 5(c)].

Reference 8 relates the increase of $I_{D}$ to a lower source access resistance due to the presence of holes (and electrons) under the source part of the recess. Even if such an effect is observed in our results [Figs. 5(a) and 5(d)], our explanation of the kink effect coincides with that of Ref. 4, confirming that the decrease of source resistance cannot be solely responsible for the kink effect.

Figure 7 shows the same profiles as those of Fig. 5 for the case of $V_{\mathrm{DS}}=3.0 \mathrm{~V}, \tau_{\mathrm{rec}}=0.1 \mathrm{~ns}$, and several values of $V_{\mathrm{GS}}$. As shown in Fig. 7(b), the number of impact ionization mechanisms decreases when $V_{\mathrm{GS}}$ is increased from $-0.3 \mathrm{~V}$ to $-0.15 \mathrm{~V}$, since the maximum electron energy is lower [Fig. 7(c)] due to the lower gate-to-drain potential. Moreover, the hole pile up is smaller, as observed in Fig. 7(a), because of both the lower number of impact ionization events and the weaker attraction of the gate potential, while the recess influence is similar in the two cases. As a consequence, the kink-related opening of the channel [Fig. 7(d)] and the associated increase of $I_{D}$ (Fig. 4) are less pronounced for $V_{\mathrm{GS}}=-0.15 \mathrm{~V}$ than for $V_{\mathrm{GS}}=-0.3 \mathrm{~V}$. For $V_{\mathrm{GS}}=0.0 \mathrm{~V}$ and $V_{\mathrm{GS}}=0.3 \mathrm{~V}$, current oscillations emerge due to Gunn effect in the gate-drain region, and the qualitative behavior of electron transport is different. Under these biasing conditions, a high-electric field domain is moving in the region between the gate and the drain. In this case, impact ionization mechanisms take place in the whole gate-drain region, especially near the drain [Fig. 7(b)], where the high electricfield domain takes the highest values when leaving the structure. Therefore, when increasing $V_{\mathrm{GS}}$ to $0.0 \mathrm{~V}$, the onset of Gunn oscillations provokes a sudden increase of impact ionization mechanisms, and also a larger pile up of holes under the source side of the gate and the recess, which leads to a stronger kink effect than for $V_{\mathrm{GS}}=-0.15 \mathrm{~V}$ (even if the gateto-drain potential is lower). Finally, for $V_{\mathrm{GS}}=0.3 \mathrm{~V}$, the attracting effect of the gate is weaker. As a result, the recess influence becomes notably more significant than that of the gate potential and the hole pile up occurs principally under

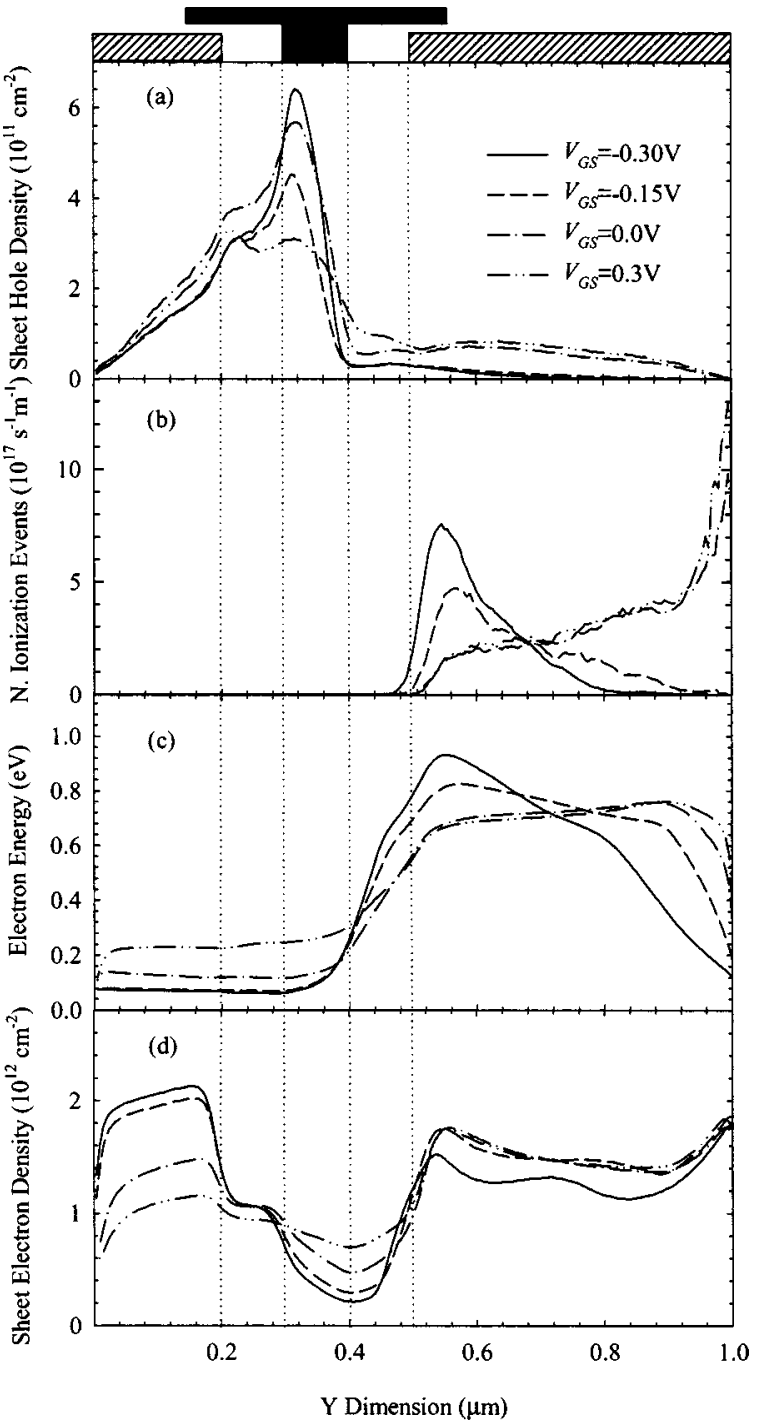

FIG. 7. Profiles along the channel of: (a) Hole sheet density, (b) impact ionization events per unit time and depth, (c) electron total energy, and (d) electron sheet density for $V_{\mathrm{DS}}=3 \mathrm{~V}, E_{\mathrm{th}}=0.8 \mathrm{eV}, S=2 \times 10^{12} \mathrm{~s}^{-1}, \tau_{\text {rec }}$ $=0.1 \mathrm{~ns}$, and different values of $V_{\mathrm{GS}}$. The position of the gate and the recess is also indicated.

the source side of the recess [Fig. 7(a)]. In this case, the amount of surface charge present at the recess is decisive for the kink behavior. ${ }^{8}$ This fact suggests that the passivation of the recess surface can be of interest when trying to reduce the kink effect for open channel conditions.

\section{CONCLUSIONS}

We have presented a microscopic analysis of kink effect in short-channel recessed $\operatorname{In}_{0.52} \mathrm{Al}_{0.48} \mathrm{As} / \mathrm{In}_{0.53} \mathrm{Ga}_{0.47} \mathrm{As}$ HEMTs based on MC simulations. The results allow to interpret the effect in terms of the pile up of holes (generated by impact ionization in the gate-drain region) in the sourcegate side of the channel. This hole pile up takes place mainly under the gate for low values of $V_{\mathrm{GS}}$, while for higher $V_{\mathrm{GS}}$ the attraction of the surface charge at the recess becomes also important as compared with that related to the gate. The positive charge due to the accumulation of holes contributes 
to open the channel and thus leads to an increase in the electron density, with the consequent enhancement in the drain current. Some practical information can be extracted from our analysis: (i) Since the accumulation of holes increases with $\tau_{\text {rec }}$, an intentional (while controlled) reduction of this time may help to avoid the appearance of kink, and (ii) the passivation of the recess surface can be of importance only for high $V_{\mathrm{GS}}$, when the effect of the surface charges on the hole accumulation is significant.

\section{ACKNOWLEDGMENTS}

This work has been partially supported by the Dirección General de Investigación (Ministerio de Ciencia y Tecnología) and FEDER through Project No. TIC2001-1754 and by the Consejería de Cultura de la Junta de Castilla y León through Project No. SA057/02.

${ }^{1}$ S. Tiwari, Compound Semiconductor Device Physics (Academic, New York, 1992).

${ }^{2}$ S. Weinreb, T. Gaier, R. Lai, M. Barsky, Y. C. Leong, and L. Samoska, IEEE Microw. Guid. Wave Lett. 9, 282 (1999).

${ }^{3}$ J. W. Archer, R. Lai, R. Grundbacher, M. Barsky, R. Tsai, and P. Reid, IEEE Microw. Wire. Compon. Lett. 11, 4 (2001).

${ }^{4}$ M. H. Somerville, A. Ernst, and J. A. del Alamo, IEEE Trans. Electron Devices 47, 922 (2000).

${ }^{5}$ W. Kruppa and J. B. Boos, IEEE Trans. Electron Devices 42, 1717 (1995).

${ }^{6}$ R. T. Webster, S. Wu, and A. F. M. Anwar, IEEE Electron Device Lett. 21, 193 (2000).
${ }^{7}$ G. Meneghesso, G. Massari, D. Buttari, A. Bortoletto, M. Maretto, and E. Zanoni, Microelectron. Reliab. 39, 1759 (1999).

${ }^{8}$ T. Suemitsu, T. Enoki, N. Sano, M. Tomizawa, and Y. Ishii, IEEE Trans. Electron Devices 45, 2390 (1998).

${ }^{9}$ A. Di Carlo, L. Rossi, P. Lugli, G. Zandler, G. Meneghesso, M. Jackson, and E. Zanoni, IEEE Electron Device Lett. 21, 149 (2000).

${ }^{10}$ A. Sleimann, A. Di Carlo, P. Lugli, and G. Zandler, IEEE Trans. Electron Devices 48, 2188 (2001).

${ }^{11}$ J. Mateos, T. González, D. Pardo, V. Hoël, and A. Cappy, Semicond. Sci. Technol. 14, 864 (1999).

${ }^{12}$ J. Mateos, T. González, D. Pardo, V. Hoël, H. Happy, and A. Cappy, IEEE Trans. Electron Devices 47, 250 (2000).

${ }^{13}$ J. Mateos, T. González, D. Pardo, V. Hoël, and A. Cappy, IEEE Trans. Electron Devices 47, 1950 (2000).

${ }^{14}$ S. Babiker, A. Asenov, N. Cameron, and S. P. Beaumont, IEEE Trans. Electron Devices 43, 2032 (1996).

${ }^{15}$ M. V. Fischetti, IEEE Trans. Electron Devices 38, 634 (1991).

${ }^{16}$ T. P. Pearsal, Appl. Phys. Lett. 36, 218 (1980).

${ }^{17}$ F. Osaka, T. Mikawa, and T. Kaneda, IEEE J. Quantum Electron. 21, 1326 (1985).

${ }^{18}$ T. Kagawa, Y. Kawamura, H. Asai, M. Naganuma, and O. Mikami, Appl. Phys. Lett. 55, 993 (1989).

${ }^{19}$ I. Watanable, T. Torikai, K. Matika, K. Fukushima, and T. Uji, IEEE Electron Device Lett. 11, 437 (1990).

${ }^{20}$ T. Brudevoll, T. A. Fjeldly, J. Baek, and M. S. Shur, J. Appl. Phys. 67, 7373 (1990).

${ }^{21}$ M. Costato and L. Reggiani, Phys. Status Solidi B 58, 471 (1973).

${ }^{22}$ K. Brennan and K. Hess, Solid-State Electron. 27, 347 (1984).

${ }^{23}$ B. Jalali and S. J. Pearton, InP HBTs: Growth, Processing, and Applications (Artech House, Boston, 1995).

${ }^{24} \mathrm{~W}$. Liu, Fundamentals of III-IV Devices (HBTs, MESFETs, and HFETs/ HEMTs (Wiley, New York, 1999). 